\title{
ANUNPRECEDENTED CASE OF COEXISTENCE OF ALPORT SYNDROME AND ANKYLOSING SPONDYLITIS
}

Thais de Campos Ferreira Pinto",*, Vanessa Félix Nascimento Coelho', Maria Veronica Russo Macchi', Lucas Eduardo Pedri', José Alexandre Mendonça', Rubens Bonfiglioli', Nadia Regina Bossolan Schincariol', Vanessa Ramos Guissa', Claudia Valeria Vierhout ${ }^{1}$, José Roberto Provenza ${ }^{1}$, André Marun Lyrio ${ }^{1}$

1.Pontifícia Universidade Católica, Campinas (SP), Brazil.

${ }^{*}$ Corresponding author: thais.fmt@hotmail.com

\section{BACKGROUND}

Alport syndrome (AS) is a rare genetic disease involving a structural defect in type IV collagen - a protein that constitutes the glomerular basal membrane. The most common subtype is an X-linked $(\mathrm{XL})$ heritage $(80 \%)$, but there are also the autosomal recessive (AR) (15\%) and the autosomal dominant (AD) kinds (5\%). Estimated prevalence is 1:10 000 for XLAS and 1:50 000 for ARAS. Once it affects the glomerular basal membrane, progressive kidney disease is one of the main features. Age of onset is anywhere from childhood to elderly age. Males are severely affected in X-linked Alport syndrome (XLAS) and present with early end stage renal disease (ESRD). Other symptoms may be sensorineural hearing loss and ocular anomalies. Clinical features, family history, renal biopsy and molecular genetic testing stablish the diagnosis. There is no cure and the treatment is only symptomatic, aiming to prevent the progression of ESRD. Therefore, the prognosis is poor and can lead to renal transplantation.

\section{CASE REPORT}

39-year-old man looked for a rheumatologist complaining of Raynaud's phenomenon that started 3 years earlier, redness of both eyes - diagnosed as bilateral anterior uveitis - and inflammatory low back pain. He also referred recent sudden hearing loss and foamy urine. This man was admitted to the emergency room a few days later with acute pulmonary edema and cardiac magnetic resonance imaging (MRI) found non-coronary myocardial fibrosis. Screening tests revealed positive HLA B27 test and bilateral grade IV sacroiliitis on X-ray. Proteinuria of $3.73 \mathrm{~g}$ was also found and renal biopsy presented with a focal segmental glomerulosclerosis (FSGS) pattern. Patient had reported that his brother died young (36 years old) and the autopsy showed edemigenic syndrome, cardiac fibrosis and renal sclerosis. The diagnosis of ankylosing spondylitis was stablished, as well as patient was referred to a geneticist for investigation of the myocardial fibrosis, FSGS and hearing loss association (after amyloidosis diagnosis was ruled out). Genetic test showed a pathogenic variant of the COL4A3 gene (related to ARAS or ADAS): Chr2: 228.173.662 - compatible with Alport syndrome.

\section{CONCLUSION}

In addition to the unusual association between Alport syndrome and spondyloarthritis, the genetic mutation seen in this case report is also unprecedented in the literature. It is known that patients usually look for their rheumatologists for challenging diagnosis, due to the complexity of the specialty. Therefore, this specialist should always be aware of rare diagnoses. 